\title{
The Data Fitting of Motor Back-to-Back Test Based on Least Square
}

\author{
Method \\ Sun Guibin ${ }^{1,2, ~ a, ~ G u o ~ F e i ~}{ }^{1, b}$ \\ ${ }^{1}$ Xiamen University of Technology, China \\ ${ }^{2}$ Fujian key laboratory of advanced design and manufacture for bus, China \\ a1464278484@qq.com, b917301508@qq.com
}

\section{Key Words: Back-to-Back test; MATLAB; Curve Fitting}

Summary: The driving motor is the direct power source of electric vehicle, and speed torque、work efficiency of motor have a great influence on the matching of vehicle powertrain. The back-to-back motor test not only can detect some key parameters, but also can detect the motor performance is good or bad. However, the original test data can't reflect the essence of the test directly, and through the fitting of test data, we can not only get the functional relationship that meets the data, but also understand mathematical and physical meaning reflected by data better. Therefore, the fitting of test data is essential. This paper is based on least squares theory, two curve fitting method in MATLAB are chosen to fit the back-to-back test data, and the analysis of fitting effect graph and fitting function shows that the two curve fitting methods can work.

\section{Introduction}

In scientific research, we often get some discrete test data which can't reflect the essence of test. Thus, it is necessary to find the approximate functional relationship between independent variable and dependent variable, and fitting the experimental data can show the regularity of the data visually and intuitively ${ }^{[1]}$.

In 《Influence of Data Fitting Times on Objective Evaluation Index of Vehicle Handling Stability $\rangle$, the accuracy of test is improved based on linear regression methods and reduction of number of fitting in order to process systematic error and random error in vehicle handling and stability test ${ }^{[2]}$. In 《A Fitting on Engine Characteristic Map Based on Text Data》, in order to optimize the engine characteristic curve, using the way of curve interpolation of operating point in different sections which contain comparison of three interpolation methods and a new method of adding interpolation data at virtual operating point to optimize the engine characteristic curve ${ }^{[3]}$. In 《Research on Uncertainty in Motor Test Data Fitting》, a mathematical model based on uncertainty theory is constructed and verified by experiments to solve the problem of abnormal existence in motor test data ${ }^{[4]}$.

In this paper, based on least squares theory, we use Polynomial and Curve Fitting Tool which contain Fourier and Rational in MATLAB to fit the data from back-to-back motor test.

\section{Back-to-Back Test}

The main function of the back-to-back test is to complete the scientific experiment and delivery test of driving motor and its controller. The relevant measuring instruments can also be used to analyze the parameters such as temperature, noise and efficiency of driving motor ${ }^{[5]}$. As 
shown in Figure 1, Left to right: motor tested torque-speed transducer、accompanying motor whose controller connect with computer, and the speed and torque input of motor tested can be controlled by related software in computer. The parameters of motor tested are shown in table 1.

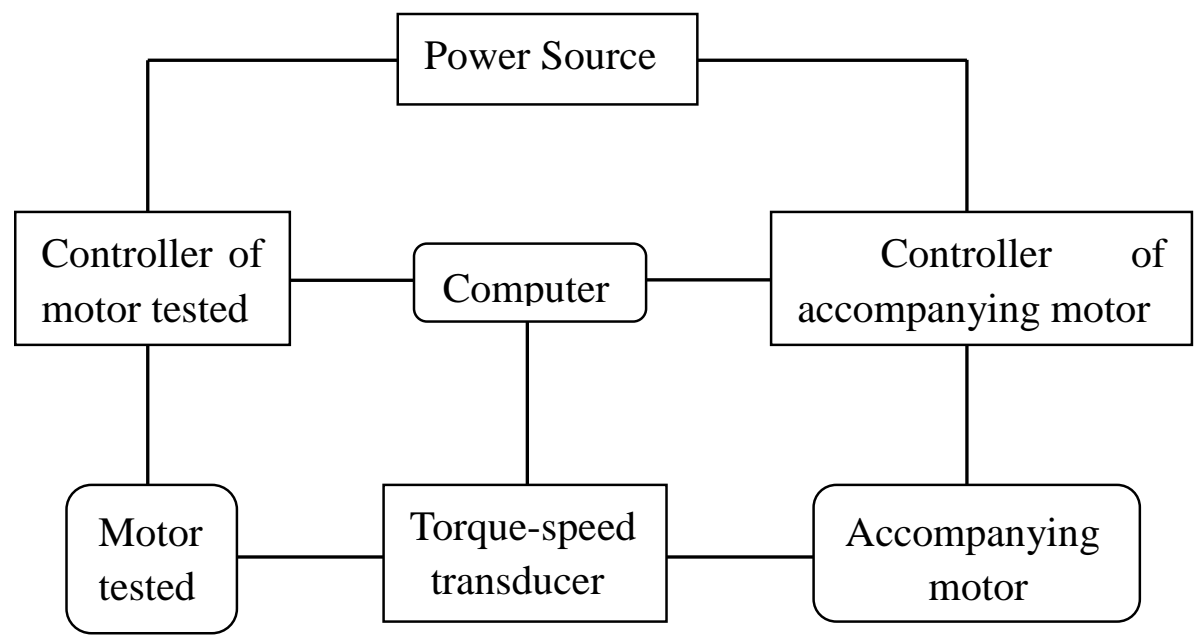

Figure 1 Motor back-to-back test bench

Table 1 The parameter of motor tested

\begin{tabular}{|c|c|c|c|}
\hline Motor Model & $\begin{array}{c}\text { Rated/Peak Torque } \\
{[\mathrm{N} . \mathrm{m}]}\end{array}$ & $\begin{array}{c}\text { Rated/Peak Power } \\
{[\mathrm{KW}]}\end{array}$ & $\begin{array}{c}\text { Maximum Speed } \\
{[\mathrm{rpm}]}\end{array}$ \\
\hline TZ445XSGL1 & $764 / 2000$ & $80 / 150$ & 3000 \\
\hline
\end{tabular}

As shown in Table 2 and Table 3, those data are got from back-to-back motor test.

Table 2 The data of line current and line voltage

\begin{tabular}{|c|l|l|l|l|l|l|l|}
\hline I[A] & 36.5 & 99.7 & 164.2 & 193.9 & 230.6 & 264.8 & 302.7 \\
\hline U[V] & 190.3 & 196.6 & 206.5 & 208.6 & 212.4 & 220.2 & 228.0 \\
\hline
\end{tabular}

Table 3 The data of speed and power input

\begin{tabular}{|c|l|l|l|l|l|l|l|l|l|l|l|}
\hline $\mathrm{n}[\mathrm{r} / \mathrm{min}]$ & 125 & 250 & 500 & 750 & 1000 & 1250 & 1500 & 1750 & 2000 & 2250 & 2500 \\
\hline $\mathrm{P}[\mathrm{kw}]$ & 20 & 45 & 94 & 132 & 178 & 179 & 183 & 183 & 183 & 182 & 178 \\
\hline
\end{tabular}

\section{Curve Fitting in MATLAB}

\section{The definition of curve fitting}

Generally speaking, the relationship of variables is not always linear, for example, the relationship between alcohol concentration and time in drunk-driving test; the relationship between effect and course of treatment in medicine. These data are scattered and can't express relationship accurately with the liner function so that these data can be expressed by fitting curves which could represent changed trend between the two variables ${ }^{[6]}$.

\section{The principal of curve fitting}

When using the curve fitting to process data, due to the influence of error, we usually adopt the least squares principle based on minimum residual sum of squares. For example, in the data set $\left\{\left(x_{i}, y_{i}\right)\right\}(i=0,1,2, \ldots, m)$ and the given function $\varphi$, solving $p(x) \in \varphi$ to make the square sum of residues $\mathrm{E}^{2}$ minimum, and $E^{2}=\sum\left[p\left(x_{i}\right)-y_{i}\right]^{2}$. Geometrically speaking, making the distance 
between the given data set $\left\{\left(x_{i}, y_{i}\right)\right\}(i=0,1,2, \ldots, m)$ and the curve $y=p(x)$ minimum. Thus, the function $p(x)$ is called the fitting function or the least square, the method of getting fitting function is called the least square method ${ }^{[7]}$.

\section{Methods of curve fitting in MATLAB}

There are two primary fitting methods in MATLAB. The first method, inputting function directly in command window of MATLAB to fit data, and it also requires acquaintance with various kinds of linear and non-linear fitting function; the other one is using the Curve Fitting Tool which is one of function of MATLAB to fit graphically, it works vividly and intuitively ${ }^{[8]}$. How to fit the data of back-to-back test by the above methods will be introduced in the following.

\section{The fitting function Polyfit}

The curve fitting in function form contains polynomial curve fitting function Polyfit and non-linear fitting function consists of lsqcurvefit lsqnonlin. When we choose the appropriate type of fitting curve, we usually start from the professional requirements of data, and also choose type from the scatter plot where we could observe the distribution of scatter. Therefore, we fit the data of linear current and linear voltage with the usage of Polyfit according to the scatter plot of data, as shown in Figure 4.

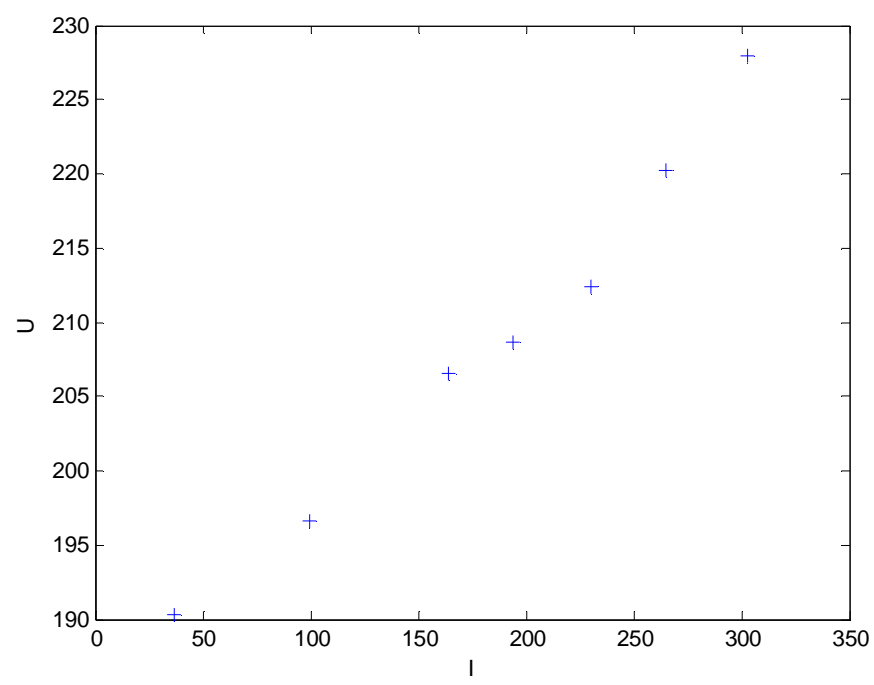

Figure 4 Scatter plot of data of line current and line voltage

Firstly, the input formats of function Polyfit, as follows.

$$
\mathrm{p}=\operatorname{polyfit}(\mathrm{x}, \mathrm{y}, \mathrm{n})
$$

$\mathrm{X}$ and $\mathrm{y}$ represent the data fitted, $\mathrm{n}$ represents the order of fitting polynomial.

For example, the above fitting polynomial turns into linear fitting in case of $n=1$.

Then, enter the following command in command window of MATLAB according to the data in table 2.

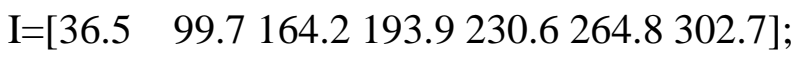

$$
\begin{aligned}
& \mathrm{U}=\left[\begin{array}{lllll}
190.3 & 196.6 & 206.5 & 208.6 & 212.4 \\
220.2 & 228.0
\end{array}\right] \text {; } \\
& \mathrm{p}=\operatorname{polyfit}(\mathrm{I}, \mathrm{U}, 2) \\
& \mathrm{U} 1=\operatorname{polyval}(\mathrm{p} 1, \mathrm{I}) \text {; } \\
& \text { plot(I,U,'+',I,U1) }
\end{aligned}
$$

The fitting result is shown in Figure 5, and the fitting polynomial is $\mathrm{U}=0.0002 \mathrm{I}^{2}+0.0691 \mathrm{I}+187.79$. 


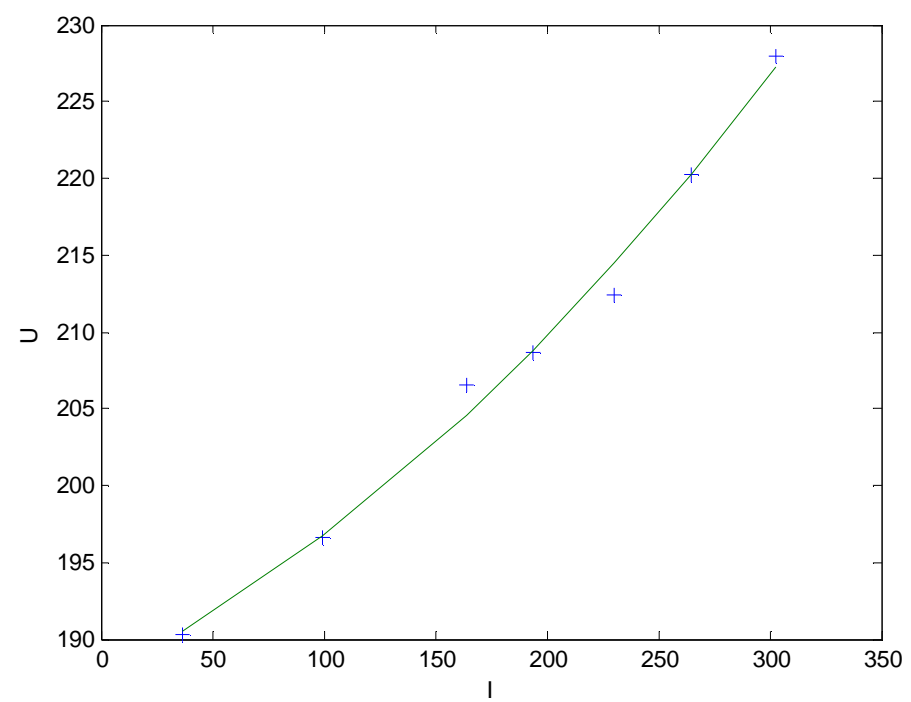

Figure 5 Fitting result of data of line current and line voltage

\section{Curve Fitting Tool}

The cftool is one of the fitting function of MATLAB, which is easy to use and provides a wealth of fitting algorithms; it also can draw scatter plot to visualize the fitting data automatically and determine the accuracy of fitting results according to residual; not only exclude abnormal data-point from the fitted curve, but also can use extrapolation, interpolation and differential to process data-point ${ }^{[9]}$. Therefore, we use the cftool to fit the data of table 3 in the following.

Firstly, enter "n=[125 250500750100012501500175020002250 2500]; P=[20 4594132 178179183183183182 178]; cftool(n,P); " in command window. Then, the operation interface of cftool will appear, which is shown in Figure 6.

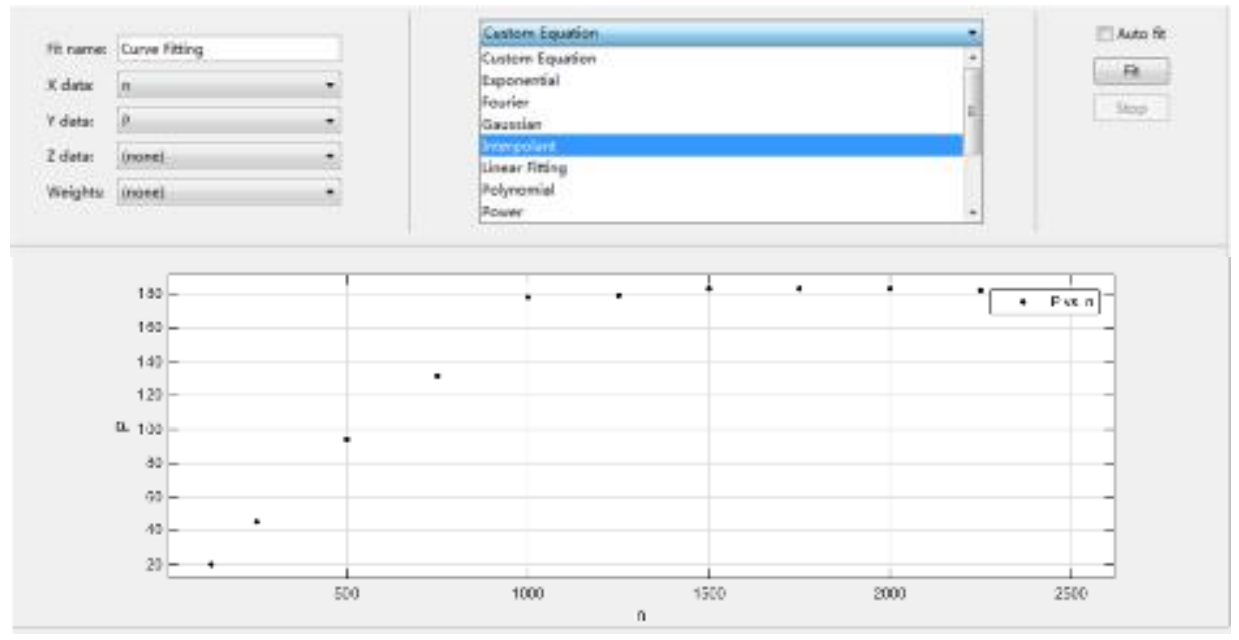

Figure 6 The operation interface of cftool

In the above Figure, "X data" and "Y data" represent the fitting data; choose the type of fitting function in the right options bar; the scatter of fitting data is at the bottom. We choose the Rational and the Fourier to fit based on the distribution of data in scatter, and we get the fitting Figures and specific fitting functions, which are shown in Figure 7 and table 4. 

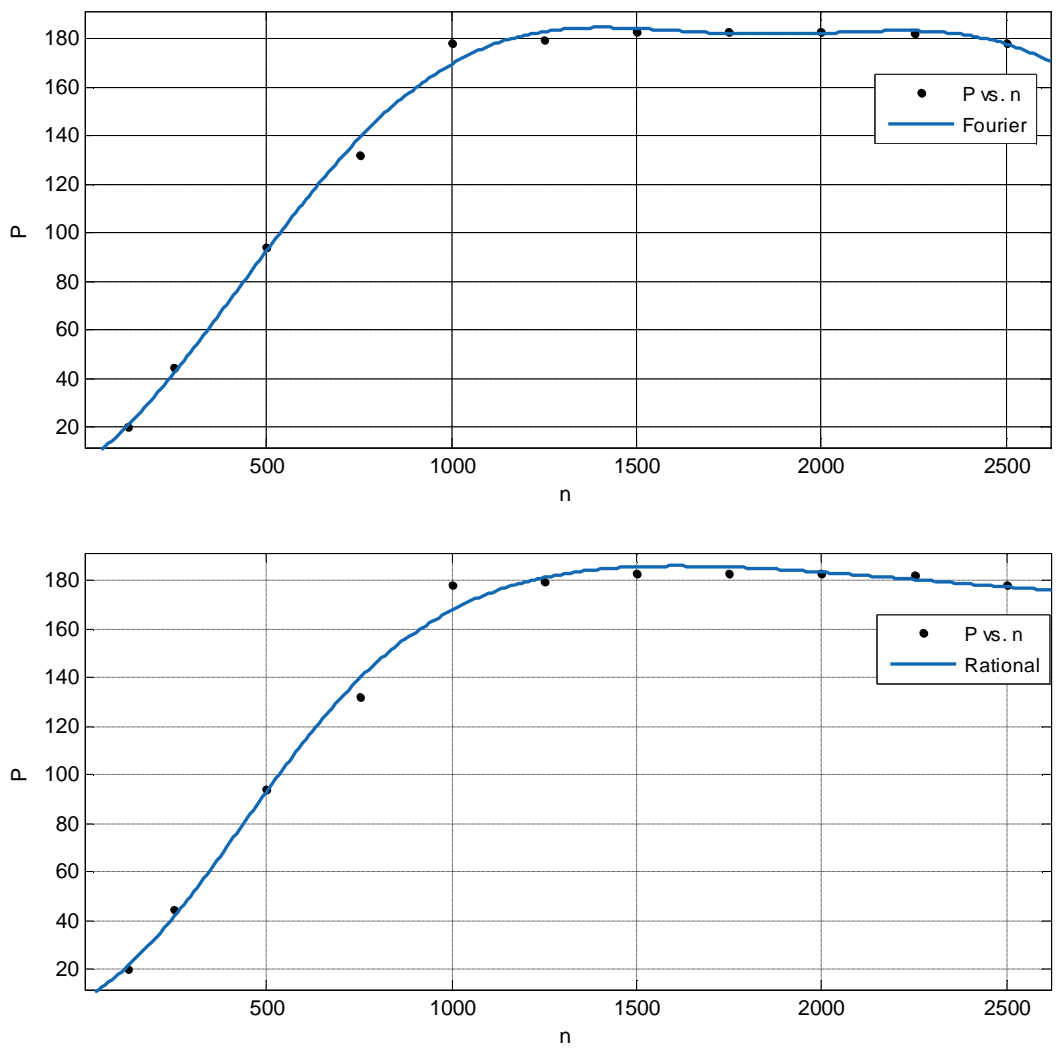

Figure 7 The fitting results of Fourier and Rational

Table 4 The specific fitting function of Fourier and Rational

\begin{tabular}{|c|c|c|c|c|}
\hline & Fitting function & coefficient & RMSE & R-Square \\
\hline Fourier & $f(x)=\frac{p_{1} * x^{2}+p_{2} * x+p_{3}}{x^{2}+q_{1} * x+q_{2}}$ & $\begin{array}{l}P_{1}=126.7 \quad P_{2}=69730 \\
P_{3}=6637000 q_{1}=-646.2 \\
q_{2}=857000\end{array}$ & 5.3549 & 0.9962 \\
\hline Rational & $\begin{array}{l}f(x)=a_{0}+a_{1} * \cos (x * w)+b_{1} * \sin (x * w) \\
+a_{2} \cos (2 x * w)+b_{2} * \sin (2 x * w)\end{array}$ & $\begin{array}{l}a_{0}=116.1 \quad a_{1}=-88.77 \\
a_{2}=-22.41 \quad b_{1}=33.29 \\
b_{2}=18.66 w=0.001529\end{array}$ & 5.6180 & 0.9949 \\
\hline
\end{tabular}

\section{Result Analysis}

In one hand, as shown in Figure 5 and 7, although most of the test data-points pass the fitting curve, there are also individual data-points for inflection point or hovering on both sides of the curve, which can be solved by the piecewise fitting function ${ }^{[10]}$. In the other hand, by comparing specific functions obtained by the two fitting methods, it can be seen that the polynomial function obtained by Polyfit is relatively simple, and it is convenient to handle the research and other aspects. However, Cftool can not only obtain the specific fitting function but also can determine the accuracy of fit based on the RMSE and other parameters. For example, in Table 4, by compare with the Fourier function, the RMSE of the Rational function is smaller and closer to 1, by which we conclude that the Rational function fits the data better. 


\section{Summary}

In this paper, two commonly used curve fitting methods in MATLAB are introduced in detail based on the two sets of experimental data measured by the motor back-to-back test based on the principle of least square method. It can be seen from the fitting results that the two fitting methods mentioned in this paper are feasible for curve fitting of variables, and from the result analysis, it is more convenient and accurate to use cftool fitting.

\section{Reference}

[1] Cheng Lizeng. Research on Principles and Applications of Curve Fitting[D]. Changsha University of Science \& Technology, 2015.

[2] Pan Jiawei, Yuan Shihai, Xie Weizhong. Influence of Data Fitting Times on Obiective Evaluation Index of Vehicle Handling Stability[J]. Automobile Technology, 2016,(2):29-32.

[3] Yang Yongcun, Xin Qingwei. A Fitting on Engine Characteristic Map Based on Text Data[J]. Journal of Naval Aeronautical and Astronautical University, 2016, 31(3):312-316.

[4] Wang Weiping, Zhao Yongbin. Research on Uncertainty in Motor Test Data Fitting[J]. Micromotors, 2011, 44(8):92-94.

[5] Wang Yanfang. Design and Manufacture of Permanent magnet Synchronous Generators[D]. Southwest Jiaotong University, 2012.

[6] Qiao Lishan, Wang Yuguang, Zeng Jinguang. Discussion on methods of curve fitting in experimental data processing[J]. Journal of Chendu University of Technology(Science \& Technology Edition), 2004, 31(1):91-95.

[7] Lu Jian. The Least Square Method And Its Application[J]. Science and Technology of West China, 2007(19):19-21.

[8] Tang Jiade. Research on Application of MATLAB in Nonlinear Curve Fitting[J]. Computer Study, 2008(1):57-59.

[9] Shi Lixin, Nie Xintian, JI Ming. Tabulated Curve Fitting Based On MATLAB Curve Fitting Toolbox [J]. New Technology \& New Process, 2007(7):39-41.

[10] Li Guanglong, Wei Zhengiun, Shangguan Wenbin. A Method for Fitting Nonlinear Experimental Data「J]. New Technology \& New Process, 2016(8):18-21.

[11] Ouvang M, Lianmin X U. Fitting method of test data based on MATLAB[J]. Journal of Nanchang Institute of Technology, 2010.

[12] Xu Liang, Song Liwei, Li Ziiian, Fu Jianfu, Wang Fuping. Saving Energy and Energy-Feedback Experimental System for Electrical Vehicle Motor[J]. Transactions of China Electrotechnical Society, 2007,22(8).

[13] Mashava A. MATLAB Curve Fitting Toolbox Model[J]. 2015.

[14] Prasanna S. Response Surface Models of Drug Interactions with Curve Fitting Toolbox[J].

[15] Esfandiari R S. Numerical Methods for Engineers and Scientists Using MATLAB ${ }^{\mathbb{B}}[\mathrm{J}]$. Crc Press, 2013.

[16] Zhang Fang, Bai Lianping, Wang Lianguo. Research on Fitting Method of Characteristic Curve of no-load Test for Asynchronous Motor[J]. Education\&Teaching Forum, 2015(9):234-236.

[17] Lin H B. Data fitting based on genetic algorithm implementation in MATLAB environment[J]. Hunan Agricultural Machinery, 2010.

[18] Song X X. A General Data Fitting Method Based on MATLAB[J]. Journal of Shanxi Datong University, 2014.

[19] Zhen Xiaozhou, Sun Wei. Performance Analysis and Modeling of Engine Based on MATLAB Fitting Toolbox[J]. Shandong Industrial Technology, 2017(18):1-2.

[20] Zhang Qi, Li ke, Zhang Chenghui. Design and Research on Characteristic Tests of PMSM for Electric Vehicle[J]. Research and Exploration in Laboratory, 2015, 34(10):47-50. 